\title{
Idiopathic CD4 lymphopenia in a case of disseminated cryptococcosis with brain, vertebral spine and reproductive organ involvement
}

\author{
Authors: Benjamin Ng Han Sim, ${ }^{A}$ Lau Yue Hui, ${ }^{A}$ Dhayalen Krishnan ${ }^{A}$ and Joyce P Joseph ${ }^{B}$
}

Cryptococcosis is an opportunistic fungal infection commonly seen in HIV cases. We present a case of disseminated cryptococcosis with multiple non-continuous infective foci in a non-HIV, non-transplant case.

KEYWORDS: Cryptococcosis, meningitis, isolated CD4 lymphopenia, HIV, non-HIV

\section{Case presentation}

A 48-year-old lady presented with headache, neck pain, fever and lethargy for 3 weeks with worsening symptoms a week prior to admission. The headache was throbbing in nature, worse in recumbent position and upon coughing. The symptoms were debilitating, hindering her work as a school teacher. Medical history was remarkable for uterine fibroid which was treated with myomectomy in 2016. She denied any high-risk behaviour, no recent travelling, no pet at home and no sick contact. Systemic review was unremarkable.

Initial assessment revealed full Glasgow coma scale and orientation; both pupils were $3 \mathrm{~mm}$ and reactive to light; neck was stiff with positive Kernig's and Brudzinki's signs; vital signs included blood pressure of $155 / 80 \mathrm{mmHg}$, heart rate at 74 beats/min and afebrile and $\mathrm{O}_{2}$ saturation was $97 \%$ under room air; and the nervous system assessment was intact without focal deficits.

Investigations showed leukocytosis $\left(19.3 \times 10^{9} / \mathrm{L}\right)$ with predominant neutrophils (76\%), elevated C-reactive protein $(57.5 \mathrm{mg} / \mathrm{L})$ and erythrocyte sedimentation rate $(56 \mathrm{~mm} / \mathrm{H})$. Her renal and liver function was within normal limit. Computed tomography (CT) of brain with contrast showed bilateral hemisphere meningeal enhancement. A diagnostic lumbar puncture was performed.

\section{Diagnosis}

Provisional diagnosis of infective meningitis was given, both ceftriaxone and acyclovir were initiated as empirical treatment. The possibility of tuberculosis (TB) meningitis and cryptococcal meningitis were considered in view of subacute presentation.

Authors: ${ }^{\text {A }}$ neurology fellow, Kuala Lumpur Hospital, Kuala Lumpur, Malaysia; ${ }^{B}$ consultant neurologist, Kuala Lumpur Hospital, Kuala Lumpur, Malaysia

\section{Initial management and prognosis}

Cerebrospinal fluid (CSF) analysis showed raised protein $(2.66 \mathrm{~g} / \mathrm{L})$, reduced CSF glucose $(0.11 \mathrm{mmol} / \mathrm{L}$ ) and raised cell count (10 cells/ microL) with negative Gram stain. Opening pressure was $48 \mathrm{~cm}$ $\mathrm{H}_{2} \mathrm{O}$. India ink staining revealed encapsulated fungi with positive cryptococcal antigen titre of 1:2560. The CSF culture grew Cryptococcus neoformans. CSF for TB workup which includes Ziehl-Neelson stain, culture and sensitivity, and polymerase chain reaction was all negative. An impression of cryptococcal meningitis was made. She was commenced on intravenous (IV) amphotericin B and IV flucytosine as per local protocol. Blood culture subsequently came back as positive for $\mathrm{C}$ neoformans hence prompted arrangement for $\mathrm{CT}$ thorax, abdomen and pelvis (TAP).

\section{Case progression and outcome}

At first week of admission, the patient's condition deteriorated with worsening headache, double vision and confusion. Examination revealed bilateral abducens nerve palsy with papilloedema. A repeated brain CT showed communicating hydrocephalus (Fig 1a). A repeated lumbar puncture showed an opening pressure of $55 \mathrm{~cm} \mathrm{H}_{2} \mathrm{O}$. Throughout admission the patient needed regular lumbar puncture to relieve her raised intracranial pressure. An extraventricular drainage was inserted and later converted to a ventriculoperitoneal shunt for persistent symptomatic raised intracranial hypertension. She completed a total of $2.9 \mathrm{~g}$ of IV amphotericin. The patient's 4-month admission was complicated with repeated episodes of nosocomial infections needing intensive care unit admission.

CT TAP showed a left adnexal mass of mixed density. There was also osteolytic lesion seen at the T12 vertebral body on the same imaging (Fig 1b and c). A biopsy of the spine lesion and excision of the left adnexal mass were performed. Both histopathological examination of the specimens came back positive for $C$ neoformans. No malignancy features were seen in the biopsies.

After a comprehensive multidisciplinary discussion between neurology, infectious disease, orthopedic and gynaecology team, a decision was made not to pursue further with operative intervention for the spine and adnexal mass. A repeated laparotomy for total abdominal hysterectomy with salpingooophorectomy was judged to be difficult given the findings of multiple fibrinous adhesions seen during the first surgical 

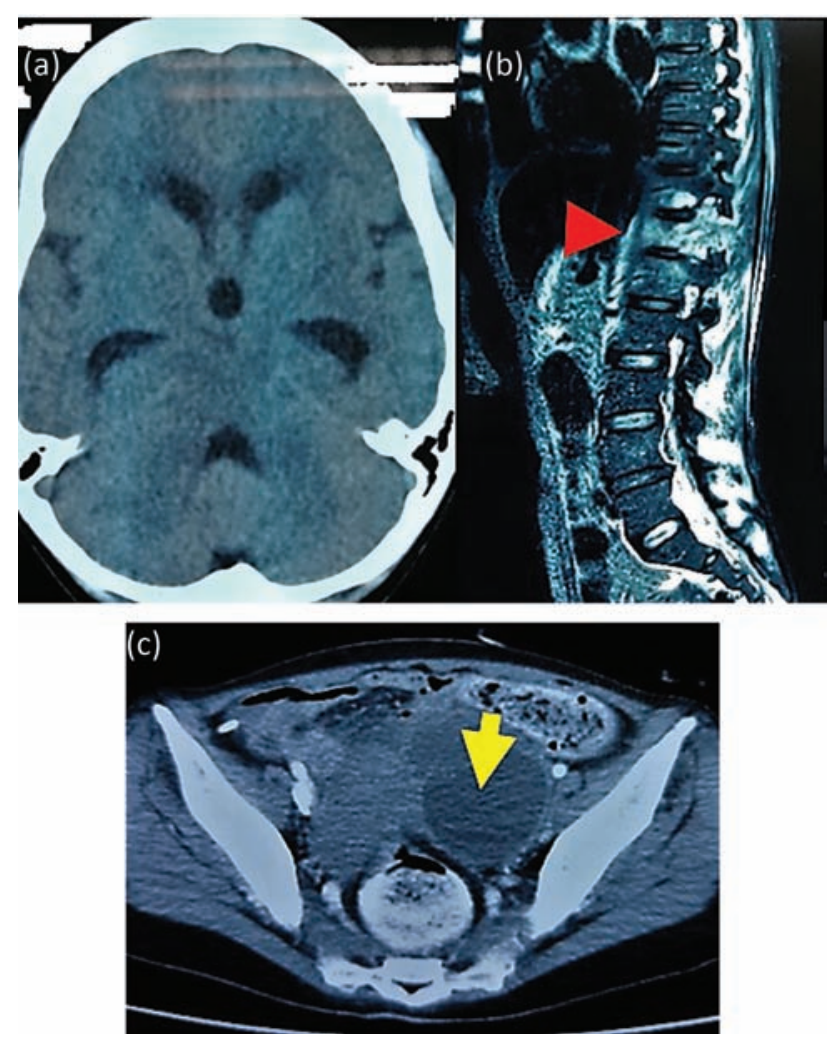

Fig 1. Computed tomography scans. (a) Communicating hydrocephalus. (b) Thoracic vertebral spine osteolytic lesion (red arrow). (c) Left adnexal mass (yellow arrow).

operation. The vertebral infective foci were not able to be excised as it was felt that operative intervention may cause the infection to disseminate. Thus, the patient was placed on lifelong maintenance of fluconazole to keep the infection dormant.

Six months after discharge, she made remarkable recovery with mild cognitive deficit. Her serial CD4 count was low with repeated negative HIV result.

\section{Discussion}

Cryptococcosis is a fungal infection caused by a cryptococcus sp. The infection usually affects HIV cases when the CD4 is less than 100 cells $/ \mu \mathrm{L}$ although non-HIV cases are seen as well. ${ }^{1}$ The sites involved include lung, brain and, less commonly, the bone and vertebral spine. Cases of disseminated cryptococcal infection defined by either the presence of Cryptococcus in the blood stream or involvement of two or more non-contiguous sites has been well described in HIV positive patient. ${ }^{2}$ However disseminated cryptococcal infection is not common among the non-HIV patients especially when the female reproductive organ is involved. ${ }^{3}$

Our case had disseminated cryptococcal infection with brain, vertebral spines and reproductive organ involvement with repeatedly negative HIV result. Another possibility would be a primary malignancy with metastasis and secondary immunosuppression predisposing to cryptococcal infection. However, biopsies pointed towards infection rather than malignancy.

A detailed review of case history showed neither immunosuppressant medication usage nor features of connective tissue disorders. The immunoglobulin levels came back as normal however the CD4 count was persistently low.

This case highlighted a rare and important predisposition for cryptococcal infection. Idiopathic CD4 lymphopenia is defined as a CD 4 count of less than 300 cells/ $\mu \mathrm{L}$ or less than $20 \%$ of all lymphocytes for two readings at least 6 weeks apart in the absence of HIV infection or other secondary causes. ${ }^{4}$ Our case's first CD4 count was 330 cells/ $\mu \mathrm{L}$ ( $11 \%$ of total lymphocytes) and the second CD 4 count was 227 cells $/ \mu \mathrm{L}$ (11\% of total lymphocytes), both taken 8 months apart.

Idiopathic CD4 lymphopenia needs to be considered in HIV negative patient with cryptococcosis as identification of this rare entity may have both therapeutic and prognostic implication. A patient with idiopathic CD4 lymphopenia who had history of cryptococcosis may warrant lifelong fungal static agent to prevent relapse. However strong evidence to answer this clinical question is still lacking. Literature review showed that CD4 count is an important prognostic factor in cryptococcal infection. One study showed that the odds ratio of contracting disseminated cryptococcal infection was 23.3 among individual with reduced CD4 count. ${ }^{5}$ Other poor prognostic markers for cryptococcosis includes positive CSF India ink, CSF white cell count less than 20 cells/microL, initial CSF or serum cryptococcal antigen titre more than 1:32 and/or high opening pressure of lumbar puncture. ${ }^{6}$ Our patient fulfilled all the poor prognostic markers identified.

\section{Key learning points}

$>$ Cryptococcosis is an opportunistic fungal infection commonly seen in HIV cases but also encountered among non-HIV cases.

> Idiopathic CD4 lymphopenia is a rare and important predisposing risk for non-HIV cryptococcosis.

> Idiopathic CD4 lymphopenia has possible therapeutic and prognostic implication in the management of non-HIV cryptococcal infection. -

\section{Acknowledgement}

The authors would like to thank the director general of Malaysia Ministry of Health and Dr Santhi Datuk Puvanarajah, head of neurology department of Kuala Lumpur Hospital for their permission and blessing to publish this case.

\section{References}

1 Jarvis JN, Harrison TS. HIV associated cryptococcal meningitis. AIDS 2007;21:2119.

2 Cox GM, Perfect JR. Cryptococcus infection outside the central nervous system. UpToDate 2017.

3 Gopal M, McCrosson S, Edmonds P, Klein T. Cryptococcosis of the upper genital tract. AIDS Patient Care STDS 2009;23:71-3.

4 Ahmad DS, Esmadi M, Steinmann WC. Idiopathic CD4 lymphopenia: Spectrum of opportunistic infections, malignancies and autoimmune diseases. Avicenna J Med 2013;3:37-47.

5 Ding Y, Li P, He Q et al. The CD4 T-lymphocyte count is an important predictor for the prognosis of cryptococcosis. Eur J Clin Microbiol Infect Dis 2017:36:897-904.

6 Diamond RD, Bennett JE. Prognostic factors in cryptococcal meningitis. A study in 111 cases. Ann Intern Med 1974;80:176.

Address for correspondence: Dr. Benjamin Ng Han Sim, Neurology Department, Kuala Lumpur Hospital, Jalan Pahang 50586 Federal Territory of Kuala Lumpur, Malaysia.

Email: drben84@gmail.com 